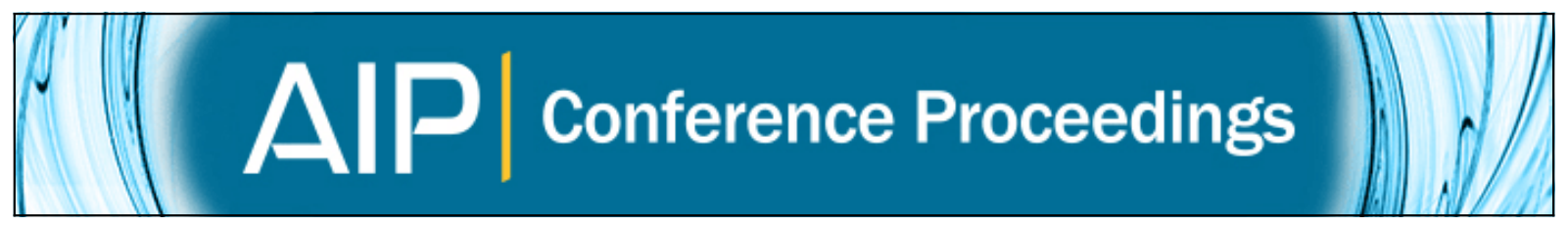

Beyond the bump-hunt: A game plan for discovering dynamical dark matter at the LHC

Keith R. Dienes, Shufang Su, and Brooks Thomas

Citation: AIP Conference Proceedings 1743, 020013 (2016); doi: 10.1063/1.4953280

View online: http://dx.doi.org/10.1063/1.4953280

View Table of Contents: http://scitation.aip.org/content/aip/proceeding/aipcp/1743?ver=pdfcov

Published by the AIP Publishing

Articles you may be interested in

Measuring properties of dark matter at the LHC

AIP Conf. Proc. 1604, 381 (2014); 10.1063/1.4883454

GUT and supersymmetry at the LHC and in dark matter

AIP Conf. Proc. 1467, 29 (2012); 10.1063/1.4742076

Hunting for dark matter in X-rays

AIP Conf. Proc. 1427, 330 (2012); 10.1063/1.3696228

Supersymmetry, Dark Matter and the LHC

AIP Conf. Proc. 1200, 145 (2010); 10.1063/1.3327553

Hunting for most of the universe-Dark matter

AIP Conf. Proc. 194, 333 (1989); 10.1063/1.38802 


\title{
Beyond the Bump-Hunt: A Game Plan for Discovering Dynamical Dark Matter at the LHC
}

\author{
Keith R. Dienes ${ }^{1,2, a)}$, Shufang $\mathrm{Su}^{1, \mathrm{~b})}$ and Brooks Thomas ${ }^{3,4, \mathrm{c})}$ \\ ${ }^{1}$ Department of Physics, University of Arizona, Tucson, AZ 85721 USA \\ ${ }^{2}$ Department of Physics, University of Maryland, College Park, MD 20742 USA \\ ${ }^{3}$ Department of Physics, Reed College, Portland, OR 97202 USA \\ ${ }^{4}$ Department of Physics, Colorado College, Colorado Springs, CO 80903 USA \\ a)dienes@email.arizona.edu \\ b)shufang@email.arizona.edu \\ c)bthomas@coloradocollege.edu
}

\begin{abstract}
Dynamical Dark Matter (DDM) is an alternative framework for dark-matter physics in which an ensemble of individual constituent fields with a spectrum of masses, lifetimes, and cosmological abundances collectively constitute the dark-matter candidate, and in which the traditional notion of dark-matter stability is replaced by a balancing between lifetimes and abundances across the ensemble. In this talk, we discuss the prospects for distinguishing between DDM ensembles and traditional dark-matter candidates at hadron colliders - and in particular, at the upgraded LHC - via the analysis of event-shape distributions of kinematic variables. We also examine the correlations between these kinematic variables and other relevant collider variables in order to assess how imposing cuts on these additional variables may distort — for better or worse — their event-shape distributions.
\end{abstract}

\section{INTRODUCTION}

Dynamical Dark Matter (DDM) $[1,2]$ is an alternative framework for dark-matter physics in which an ensemble of individual constituent fields with a spectrum of masses, lifetimes, and abundances collectively constitute the darkmatter candidate, and in which the traditional notion of dark-matter stability is replaced by a balancing between lifetimes and abundances across the ensemble. In these proceedings, we discuss the prospects for distinguishing between DDM ensembles and traditional dark-matter candidates at hadron colliders. Simple "bump-hunting" searches are typically insufficient for this purpose; rather, an analysis of the full distributions of event-shape variables is required. Distribution-based searches of this sort are particularly subtle because cuts imposed on the data for the purpose of background reduction can distort event-shape distributions in unexpected ways, thereby obscuring signals of new physics. Nevertheless, they can serve to distinguish DDM ensembles from traditional dark-matter candidates.

One class of DDM scenarios for which this analysis strategy can be particularly fruitful are those wherein the ensemble constituents are produced via the decays of additional, heavier "parent" particles which are charged under $S U(3)_{c}$ color and can therefore be pair-produced copiously at hadron colliders. For example, in cases in which each parent particle decays to a single ensemble constituent and a pair of hadronic jets, we find that the invariant-mass distribution of these two jets could provide a distinctive signal of Dynamical Dark Matter [3]. Similarly, in cases in which the parent particle decays primarily into a single ensemble constituent and a single jet, we find that the $M_{T 2}$ distribution can likewise provide such a signal [4]. In these proceedings, we discuss the prospects for observing a signal in each of these two cases at the upgraded LHC. In addition, we also examine the correlations between the kinematic variable used in the event-shape analysis and other relevant kinematic variables in order to assess how imposing cuts on these additional variables may distort the event-shape distribution in question. All of these results are discussed in more detail in Refs. [3, 4]. 


\section{DISTINGUISHING DDM ENSEMBLES USING KINEMATIC DISTRIBUTIONS}

As discussed above, the analysis of event-shape distributions can be particularly fruitful for distinguishing DDM ensembles in scenarios in which the ensemble constituents are produced via the decays of some additional, stronglyinteracting parent particle $\psi$. We begin by providing a concrete example of a scenario in which such an analysis could yield potentially yield a statistically significant signal of dark-sector non-minimality within the first $30 \mathrm{fb}^{-1}$ of integrated luminosity at the upgraded LHC. In particular, let us consider the case in which $\psi$ is a color-octet fermion and the constituent particles $\chi_{n}$ of the DDM ensemble to be color-singlet fermions which are also singlets under the rest of the SM gauge group. Moreover, let us also assume that the decay width $\Gamma_{\psi}$ of $\phi$ is dominated by contributions from an effective interaction Lagrangian of the form

$$
\mathcal{L}_{\mathrm{eff}}=\sum_{n}\left[\frac{c_{n}}{\Lambda^{2}}\left(\bar{q}_{i} t_{i j}^{a} \psi^{a}\right)\left(\bar{\chi}_{n} q_{j}\right)+\text { h.c. }\right]
$$

where $q$ denotes a SM quark, where $\Lambda$ is the cutoff scale of the effective theory, where $c_{n \alpha}$ is the dimensionless operator coefficient associated with $O_{n}^{(\alpha)}$, where $t_{i j}^{a}$ is the generator of $S U(3)$ in the fundamental representation, where the indices $i$ and $j$ label the states in the fundamental representation, and where $a$ labels the states in the adjoint representation. These contributions give rise to three-body decays of the form $\psi \rightarrow j j \chi_{n}$, where $j$ denotes a hadronic jet. Finally, we shall assume that the operator coefficients $c_{n}$ scale according to a power-law of the form

$$
c_{n}=c_{0}\left(\frac{m_{n}}{m_{0}}\right)^{\gamma} \text {, }
$$

where $c_{0}$ is the operator coefficient of the lightest ensemble constituent and where $\gamma$ is a general power-law index. Both $c_{n}$ and $\gamma$ are taken to be free parameters of the model. Moreover, we shall assume a mass spectrum of the form

$$
m_{n}=m_{0}+n^{\delta} \Delta m,
$$

where $m_{0}$ is the mass of the lightest ensemble constituent and where the dimensionless index $\delta$ and the mass-splitting parameter $\Delta m$ are likewise taken to be free parameters. In what follows, we shall adopt the convention that $\Delta m>0$ and $\delta>0$ in order to ensure that $m_{n}$ increases monotonically with $n$.

The event-shape distribution on which we shall focus with regard to this DDM scenario is the invariant-mass distribution of the two hadronic jets produced via the decay of a single $\psi$ particle. The differential partial width for the decay process $\psi \rightarrow j j \chi_{n}$ with respect to the invariant mass $m_{j j}$ into a final state involving a particular ensemble constituent $\chi_{n}$ is found to be [3]

$$
\frac{d \Gamma_{\psi n}}{d m_{j j}}=\frac{c_{n}^{2} m_{j j} \sqrt{m_{\psi}^{4}-2 m_{\psi}^{2}\left(m_{j j}^{2}+m_{n}^{2}\right)+\left(m_{j j}^{2}-m_{n}^{2}\right)}}{96(2 \pi)^{3} m_{\psi}^{3} \Lambda^{4}}\left[m_{\psi}^{4}+m_{\psi}^{2}\left(m_{j j}^{2}-2 m_{n}^{2}\right)-2 m_{j j}^{4}+m_{j j}^{2} m_{n}^{2}+m_{j j}^{4}\right] .
$$

The corresponding partial width for $\psi \rightarrow j j \chi_{n}$ is

$$
\Gamma_{\psi n}=\frac{c_{n}^{2}}{384(2 \pi)^{3} \Lambda^{4} m_{\psi}^{3}}\left[m_{\psi}^{8}-8 m_{\psi}^{2} m_{n}^{2}\left(m_{\psi}^{4}-m_{n}^{4}\right)+12 m_{\psi}^{4} m_{n}^{4} \ln \left(\frac{m_{n}^{2}}{m_{\psi}^{2}}\right)\right] .
$$

Furthermore, since we are assuming decays of this sort dominate the total width $\Gamma_{\psi}$ of $\psi$, this total width is simply $\Gamma_{\psi} \equiv \sum_{n} \Gamma_{\psi n}$. The corresponding total differential dijet invariant-mass distribution is sum of the $m_{j j}$ distributions for each individual decay channel, as given by Eq. (4), each weighted by the corresponding decay branching $\mathrm{BR}_{\psi n} \equiv$ $\Gamma_{\psi n} / \Gamma_{\psi}$ :

$$
\frac{1}{\Gamma_{\psi}} \frac{d \Gamma_{\psi}}{d m_{j j}}=\sum_{n=0}^{n_{\max }}\left(\frac{1}{\Gamma_{\psi n}} \frac{d \Gamma_{\psi n}}{d m_{j j}} \times \mathrm{BR}_{\psi n}\right),
$$

where $n_{\max }$ is the value of $n$ corresponding to the heaviest $\chi_{n}$ kinematically accessible in $\psi$ decay.

The invariant-mass distributions associated with a variety of different DDM parameter choices in this scenario are displayed in Fig. 1. It is evidents from the figure that when $\delta$ (or $\Delta m$ ) is large and the mass-splittings between 

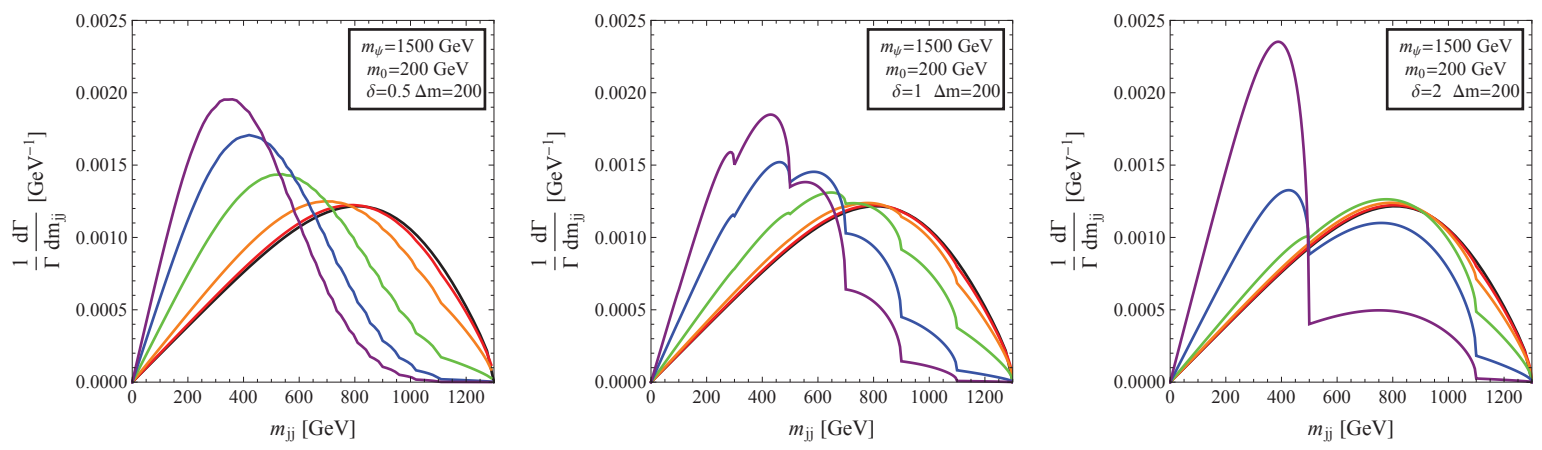

FIGURE 1. Invariant-mass distributions for the DDM ensembles considered here, shown for increasing $\delta$ with fixed $m_{\psi}=$ $1500 \mathrm{GeV}, m_{0}=200 \mathrm{GeV}$, and $\Delta m=200 \mathrm{GeV}$. The left, center, and right panels show results for $\delta=0.5, \delta=1$, and $\delta=2$, respectively. In each panel, the red, orange, green, blue, and purple curves correspond to $\gamma=\{-2,-1,0,1,2\}$, respectively, while the black curve shows the result for a traditional dark-matter candidate with $m_{\chi}=m_{0}$.

the $\chi_{n}$ are significant, individual mass edges from two or more of the $\chi_{n}$ can be resolved. By contrast, when $\delta$ (and $\delta m)$ are small, features associated with the individual $\chi_{n}$ cannot be resolved and no mass edge whatsoever is apparent; however the overall $m_{j j}$ distribution assumes a characteristic "bell" shape which nevertheless distinguishes it from the $m_{j j}$ distributions associated with traditional dark-matter candidates.

Our primary aim is to determine to degree the degree to which the characteristic invariant-mass distribution associated with a particular DDM ensemble cannot be reproduced by any traditional DM model, regardless of the mass $m_{\chi}$, spin, or other properties of the dark-matter candidate. Thus, in order to quantify the statistical significance with which any such $m_{j j}$ distribution is is truly distinctive is this sense, we adopt the following procedure. We canvass the range $0<m_{\chi}<m_{\psi}$, with a finite step size and evaluate the $m_{j j}$ distribution for each value of $m_{\chi}$ included in our survey. We then compare this distribution with the $m_{j j}$ distribution associated with the DDM model in question. In particular, we partition the range of allowed dijet invariant masses $m_{j j}^{(\min )} \leq m_{j j} \leq m_{\psi}-m_{0}$ into $n_{b}$ bins, where $m_{j j}^{(\min )}$ is some particular invariant-mass threshold imposed in order to eliminate potential issues with low statistics in bins with small $m_{j j}$. The width of each bin is taken to be equal to the dijet invariant-mass resolution $\Delta m_{j j}$ at the minimum $m_{j j}$ in the bin. This invariant-mass resolution is dominated by the uncertainty $\Delta E_{j}$ in the measurement of the energies $E_{j}$ of the individual jets; hence we take $\left(\Delta m_{j j}\right) / m_{j j} \approx\left(\Delta E_{j}\right) / E_{j}$. Moreover, since we are particularly interested in the prospects for distinguishing DDM ensembles at the LHC, we take $\left(\Delta m_{j j}\right) / m_{j j}$ to be equal to the jet-energy resolution for the ATLAS detector [5]. The jet-energy resolution for the CMS detector $[6,7]$ is quite similar.

For each value of $m_{\chi}$ in our survey, we compare the resulting $m_{j j}$ distribution with the corresponding distribution for the particular DDM model under study. As our measure of the goodness of fit in each case, we adopt the $\chi^{2}$ statistic

$$
\chi^{2}\left(m_{\chi}\right)=\sum_{k} \frac{\left[X_{k}-\mathcal{E}_{k}\left(m_{\chi}\right)\right]^{2}}{\sigma_{k}^{2}},
$$

where the index $k$ labels the bin, $X_{k}$ is the expected population of events in bin $k$ in the DDM model, $\mathcal{E}_{k}\left(m_{\chi}\right)$ is the expected population of events in bin $k$ in a traditional dark-matter model in which the dark-matter particle has mass $m_{\chi}$, and $\sigma_{k}^{2} X_{k}\left(1-X_{k} / N_{e}\right)$ is the variance in $X_{k}$ due to statistical uncertainties. The minimum value

$$
\chi_{\min }^{2} \equiv \min _{m_{\chi}}\left\{\chi^{2}\left(m_{\chi}\right)\right\}
$$

from among these individual $\chi^{2}\left(m_{\chi}\right)$ values represents the degree to which the $m_{j j}$ distribution associated with the DDM ensemble under study can be distinguished from the most similar $m_{j j}$ distribution obtained for any traditional dark-matter candidate. Thus, we adopt $\chi_{\min }^{2}$ as our final measure of the distinctiveness of the $m_{j j}$ distribution associated with this DDM model. Finally, in order to render the interpretation of these results more straightforward, we determine an effective statistical significance a given $\chi_{\min }^{2}$ value by comparing it to a $\chi^{2}$ distribution with $n_{b}-1$ degrees of freedom, computing the corresponding $p$-value, and assessing the number of standard deviations away from the mean to which the same $p$-value would correspond for a Gaussian distribution. 

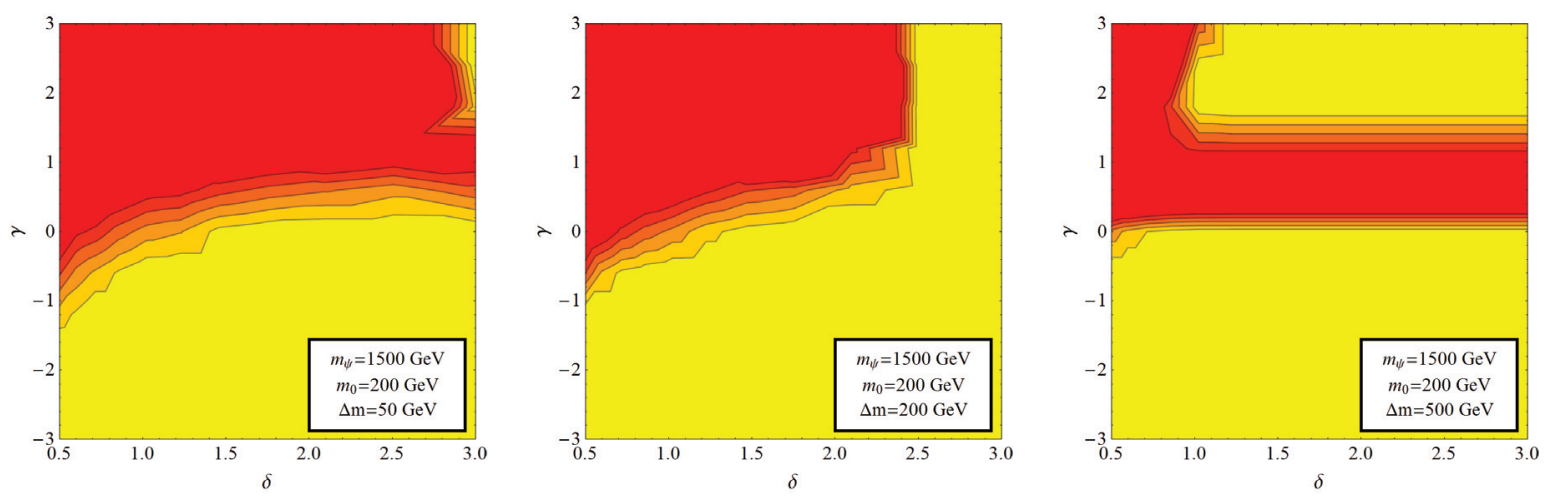

Significance:
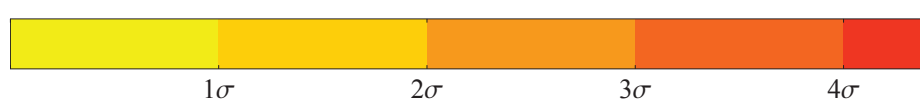

FIGURE 2. Contour plots showing the minimum significance level at which the $m_{j j}$ distribution predicted in the DDM model characterized by the parameters $m_{\psi}, m_{0}, \Delta m, \delta$, and $\gamma$ can be differentiated from the $m_{j j}$ distribution predicted in any traditional dark-matter model. In the panels shown, we have fixed $m_{\psi}=1500 \mathrm{GeV}$ and $m_{0}=200 \mathrm{GeV}$, and taken $m_{j j}^{\text {(min) }}=200 \mathrm{GeV}$ as our minimum $m_{j j}$ threshold. The results shown in each panel correspond to a signal-event count of $N_{e}=1000$, events; the left, center, and right panels of the figure show results for $\Delta m=\{50,200,500\} \mathrm{GeV}$, respectively.

In Fig. 2, we show how the significance of differentiation varies as a function of $\delta$ and $\gamma$ with $\Delta m, m_{0}$, and $m_{\psi}$ held fixed. In all of the panels shown, we have set $m_{\psi}=1500 \mathrm{GeV}$ and $m_{0}=200 \mathrm{GeV}$, and we have taken $m_{j j}^{\text {(min) }}=200 \mathrm{GeV}$ as our minimum $m_{j j}$ threshold and a step size of $100 \mathrm{GeV}$ in our scan over $m_{\chi}$. The results shown in each panel correspond to a signal-event count of $N_{e}=1000$ events, which corresponds to an integrated luminosity of roughly $\mathcal{L}_{\text {int }} \approx 30 \mathrm{fb}^{-1}$ for pair-production of a color-octet parent particle with this value of $m_{\psi}$ at $\sqrt{s}=14 \mathrm{TeV}$. The left, center, and right panels panel show the results for $\Delta m=\{50,200,500\} \mathrm{GeV}$, respectively.

The results shown in Fig. 2 indicate that throughout a substantial region of parameter space, it is possible to distinguish our toy DDM model from traditional dark-matter scenarios at a $5 \sigma$ significance level or higher. The most favorable region is that in which $\gamma \gtrsim 0.5$. The dependence on $\gamma$ is due to the fact that the branching fractions $\mathrm{BR}_{\psi n}$ of $\psi$ to the heavier $\chi_{n}$ are proportionally larger for $\gamma>0$. Decays to these heavier $\chi_{n}$ increasingly dominate the width of $\psi$ as $\gamma$ increases, and as a result, the invariant-mass distribution for the ensemble typically becomes more distinctive. By contrast, for smaller values of $\gamma$, the branching fractions to the heavier modes are suppressed, and the $m_{j j}$ distribution for the ensemble resembles that of a traditional dark-matter model with $m_{\chi}=m_{0}$. An exception to this rule occurs when $\Delta m$ and $\gamma$ are both large and the width of $\psi$ is dominated by decays to $\chi_{1}$ rather than $\chi_{0}$, resulting in an $m_{j j}$ distribution with resembles that of a traditional dark-matter candidates with $m_{\chi}=m_{1}$. The dependence of the significance of differentiation on $\Delta m$ and $\delta$ arises due to the fact that the interplay between these parameters controls the mass splittings between the different ensemble constituents and thus determines the number of $\chi_{n}$ which are kinematically accessible in $\phi$ decay. When both of these parameters are small, a significant multiplicity of $\chi_{n}$ are kinematically accessible; however, as $\Delta m$ and $\delta$ increase, the number of $\chi_{n}$ into which $\psi$ can decay decreases and, as a result, the $m_{j j}$ distribution increasingly resembles that of a traditional dark-matter candidate with $m_{\chi}=m_{0}$.

\section{CUTS AND CORRELATIONS IN DISTRIBUTION-BASED SEARCHES}

As discussed in the Introduction, correlations between kinematic variables can have profound effects on distributionbased searches for DDM ensembles. In order to demonstrate the role these effects can play in searches for dark-sector non-minimality, we provide a concrete example of a DDM scenario in which correlations have a significant impact on the prospects for distinguishing dark-sector non-minimality at the LHC. In this scenario, we shall once again take the constituent particles $\chi_{n}$ to be color-singlet fermions with a mass spectrum of the form given in Eq. (3). However, we shall take the parent particle $\psi$ to be a color-triplet scalar. Furthermore, we shall also assume that $\phi$ couples to the $\chi_{n}$ 
via the interaction Lagrangian

$$
\mathcal{L}_{\text {int }}=\sum_{n=0}^{N} \sum_{q}\left[c_{n} \psi^{\dagger} \bar{\chi}_{n} u_{R}+\text { h.c. }\right]
$$

where $u_{R}$ denotes a right-handed up quark, where the $c_{n}$ are dimensionless coupling coefficients, and where $N$ is the number of $\chi_{n}$ kinematically accessible in $\psi$ decays. Once again, we shall assume that the $c_{n}$ scale with $n$ according to a power law of the form given in Eq. (2), where $\gamma$ and $c_{0}$ are taken to be free parameters. We also assume that the decay width of $\psi$ is dominated by decay processes of the form $\phi \rightarrow q \bar{\chi}_{n}$ to such an extent that all other contributions to that width can safely be neglected, and and that all such decay processes occur promptly within the detector.

Other than variables that characterize the angular size and substructure of the two jets (considerations which are not particularly relevant for this analysis), events in the dijet $+Z_{T}$ channel are completely characterized by only six independent degrees of freedom: the six components of the momenta $\vec{p}_{1}$ and $\vec{p}_{2}$ of the jets $j_{1}$ and $j_{2}$, respectively. A number of kinematic variables can be constructed from these six degrees of freedom; however, in what follows, we shall focus on those which are found [4] to be particularly sensitive to the structure of the dark sector, including:

- The magnitude $H_{T}$ of the missing transverse momentum in the event.

- The standard $M_{T 2}$ variable [8].

In addition, our analysis must incorporate a number of additional variables which are not necessarily particularly sensitive to the structure of the dark sector, but which are useful for background reduction. Indeed the contribution to the total event rate in the dijet $+Z_{T}$ channel from SM background processes is quite significant; thus, in order to have any hope of detecting a signal of dark matter at all, stringent cuts must be imposed on the data in order to reduce these backgrounds. A minimum cut on $\not_{T}$ is generally useful for reducing these SM backgrounds, as are cuts on the following variables:

- The scalar sum $H_{T_{i j}}$ of the transverse jet momenta $p_{T_{1}}$ and $p_{T_{2}}$.

- $\quad$ The variable $\alpha_{T} \equiv p_{T_{2}} / m_{j j}[9,10]$, where $m_{j j}$ is the invariant mass of $j_{1}$ and $j_{2}$.

The latter variable is effectively a measure of the degree to which the two leading jets in the event are back-to-back. A minimum cut on this variable on the order of $\alpha_{T} \gtrsim 0.55$ has been shown to be extremely effective in reducing the sizable background from QCD processes [11, 12].
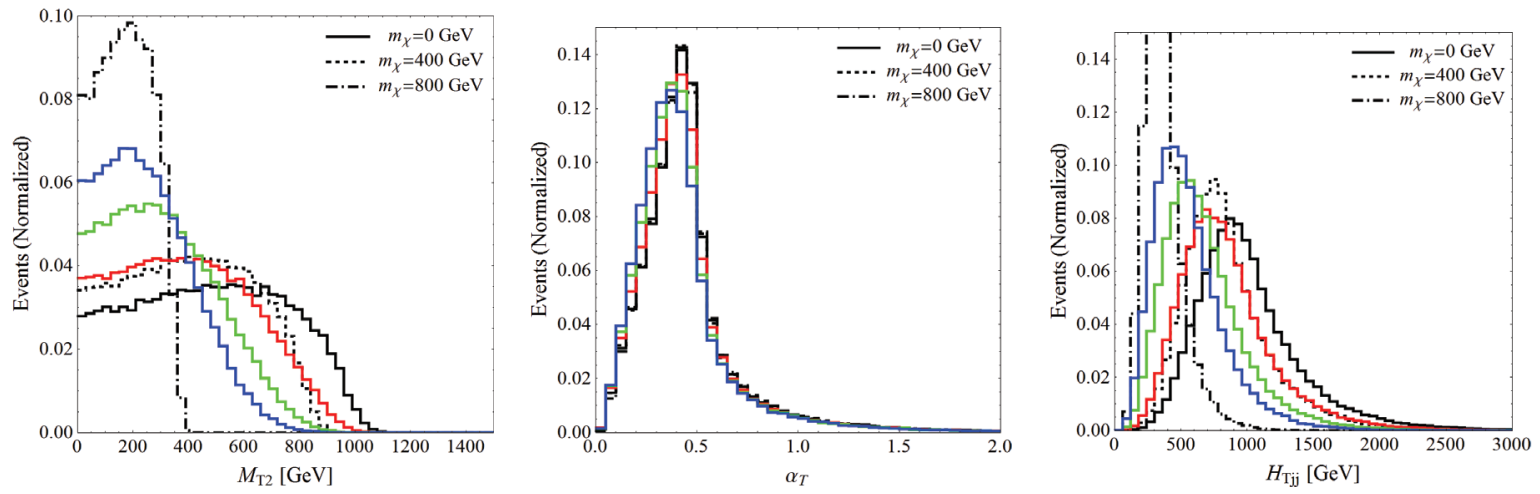

FIGURE 3. A comparison of the normalized $M_{T 2}$ (left panel), $\alpha_{T}$ (center panel), and $H_{T_{j j}}$ (right panel) distributions associated with traditional dark-matter candidates as well as DDM ensembles. In each panel, the black curves correspond to distributions for a representative set of traditional dark-matter candidates, while the colored curves in the left, middle, and right panels correspond to the DDM parameter choices $m_{\psi}=1 \mathrm{TeV}, m_{0}=200 \mathrm{GeV}, \delta=1$, and $\Delta m=\{50,300,500\} \mathrm{GeV}$, respectively, with $\gamma=0$ (red), $\gamma=1$ (yellow), and $\gamma=2$ (blue). The $M_{T 2}$ distribution shown corresponds to a trial mass $\tilde{m}=0$.

All data sets used in this study were generated at the parton level using MadGraph 5/MadEvent 1.4.8 [13] with model files obtained from the FeynRules [14] package as inputs The effects of detector uncertainties were accounted for by smearing the values for the magnitude $p_{T}$ of the transverse momentum, the azimuthal angle $\phi$, and the pseudorapidity $\eta$ obtained for each jet in the parton-level data set by Gaussian smearing functions with widths equal to the jet- $p_{T}$, pseudorapidity, and azimuthal-angle resolutions of the CMS detector $[15,16]$, respectively. In addition, 
we also incorporate a set of precuts into our analysis in order to mimic a realistic detector acceptance for the LHC detectors. In particular, we require that the two highest- $p_{T}$ jets in the event each satisfy $p_{T_{j}} \geq 40 \mathrm{GeV}$ and $\left|\eta_{j}\right| \leq 3$, and that the separation $\Delta R_{j j} \equiv \sqrt{\left(\Delta \eta_{j j}\right)^{2}+\left(\Delta \phi_{j j}\right)^{2}}$ between those two jets in $(\eta, \phi)$ space is $\Delta R_{j j} \geq 0.4$.

In Fig. 3, we display the event-shape distributions for $M_{T 2}$ (left panel), $\alpha_{T}$ (center panel), and $H_{T_{j j}}$ associated with a number of traditional dark-matter models characterized by different values of $m_{\chi}$, as well as the distributions associated with a number of DDM ensembles characterized by different values of $\gamma$ for fixed $m_{0}=100 \mathrm{GeV}, \Delta m=$ $50 \mathrm{GeV}$, and $\delta=1$. Note that these distributions have been normalized so that the total area under each is unity. Note also that all of the $M_{T 2}$ distributions shown in the figure correspond to a trial mass $\widetilde{m}=0$. This choice of $\widetilde{m}$ generally yields the most distinctive distributions because the range of possible $M_{T 2}$ values narrows as $\widetilde{m}$ is increased from zero.

The results shown in the left panel of Fig. 3 indicate that the event-shape distribution of the $M_{T 2}$ variable is particularly sensitive to the structure of the dark sector. Indeed, we find [4] that this variable typically provides the best prospects for distinguishing DDM ensembles in this channel at the LHC. By contrast, the results shown in the center panels suggest that the event-shape distribution of the $\alpha_{T}$ variable is not particularly sensitive to the structure of the dark sector and therefore not particularly useful for distinguishing among different dark-matter scenarios. The results shown in the right panel indicate that the event-shape distribution of $H_{T_{j j}}$ also displays some sensitivity to the structure of the dark sector. This suggests that care must be taken when imposing cuts on this variable for the purpose of background reduction, since such cuts may affect the data arising in different dark-matter models very differently. Indeed, this is merely a particularly striking example of how cuts imposed on one collider variable may have a significant impact on the event-shape distributions of others - a consideration which we now discuss in greater detail.
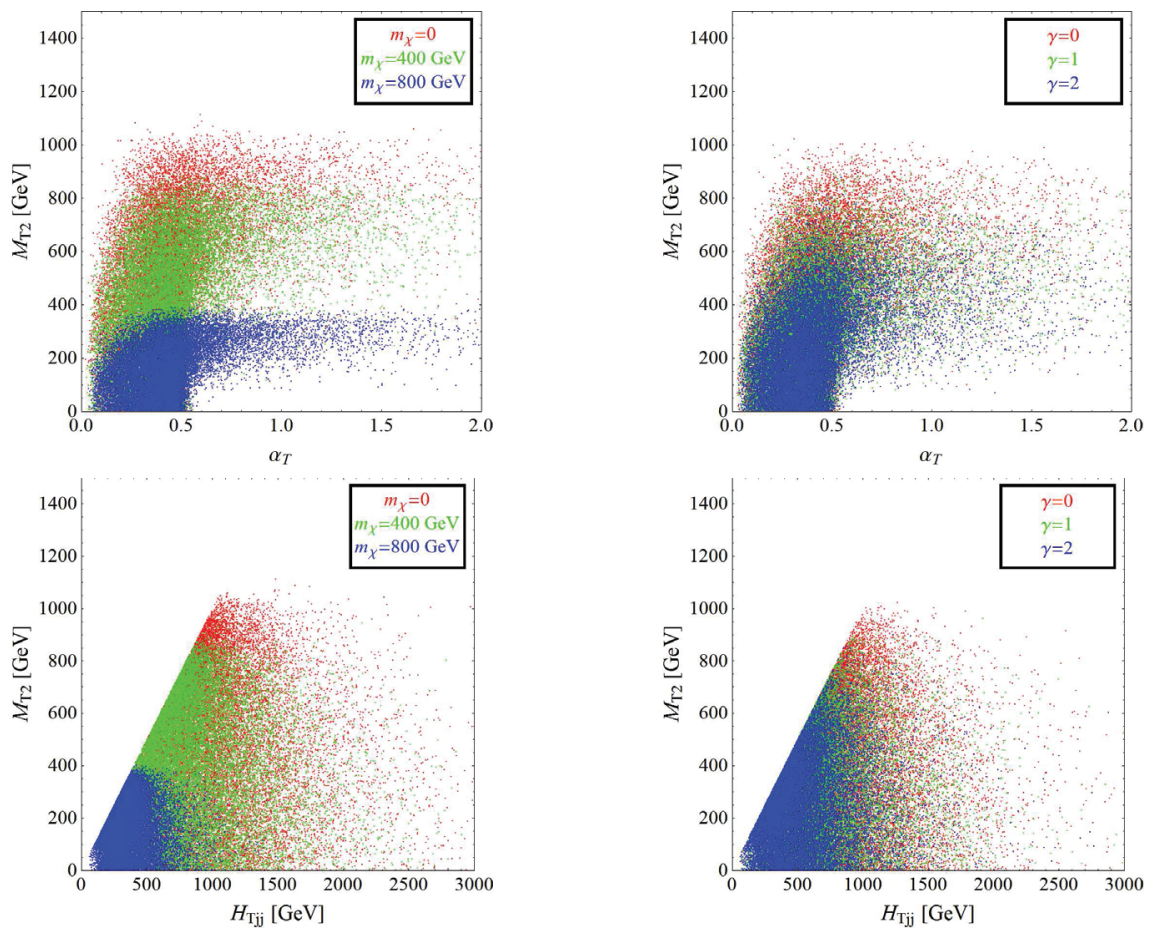

FIGURE 4. Scatter plots illustrating the correlations between $M_{T 2}$ and the selection variables $\alpha_{T}$ (top row) and $H_{T_{j j}}$ (bottom row) for a trial mass $\widetilde{m}=0$. The left panel in each row shows the results for traditional dark-matter models with $m_{\chi}=0$ (red), $m_{\chi}=400 \mathrm{GeV}$ (green), and $m_{\chi}=800 \mathrm{GeV}$ (blue), while the right panel in each row shows the results for three DDM models, with $m_{0}=100 \mathrm{GeV}, \Delta m=50 \mathrm{GeV}, m_{\phi}=1 \mathrm{TeV}$, and $\gamma=0$ (red), $\gamma=1$ (green), and $\gamma=2$ (blue).

In order to explore this issue further, we turn to directly examine the correlations between the variables $\alpha_{T}$ and $H_{T i j}$, which are important background reduction, and the $M_{T 2}$ variable, which is important for distinguishing between minimal and non-minimal dark sectors. These correlations are illustrated in the scatter plots displayed in Fig. 4 for a benchmark set of traditional dark-matter models (left column) and DDM ensembles (right column) — again for a 
trial mass $\widetilde{m}=0$. Each data point in each panel corresponds to a single event chosen randomly from the Monte-Carlo data sample for that model: its color indicates the model with which it is associated and its coordinates indicate the values $x$ and $y$ of the two kinematic variables $X$ and $Y$ of interest for the corresponding event. Thus, the density of points within the region $(x, y)$ to $(x+\delta x, y+\delta y)$ indicates the relative likelihood of values for $X$ and $Y$ within that range occurring in combination.

The results shown in Fig. 4 clearly illustrate that non-trivial correlations exist between both $\alpha_{T}$ and $H_{T_{j j}}$ and $M_{T 2}$. In particular, we see that imposing a minimum cut of the form $H_{T_{j j}}>H_{T_{j i}}^{\min }$ will preferentially select events associated with decays to lighter $\chi_{n}$, thereby effectively washing out contributions to the $M_{T 2}$ distribution from the heavier ensemble constituents that could serve as a signal of DDM. By contrast, a cut of the form $\alpha_{T}>\alpha_{T}^{\min }$ on $\alpha_{T}$ has the opposite effect: while such a cut reduces the total number of signal events, it also results in a segregation of the surviving data points corresponding to different value of $m_{n}$ into different ranges of $M_{T 2}$. This implies that cuts on $\alpha_{T}$ are not only effective in reducing SM backgrounds, but can also serve to amplify distinctions between the shapes of the $M_{T 2}$ distributions associated with different dark-matter models.
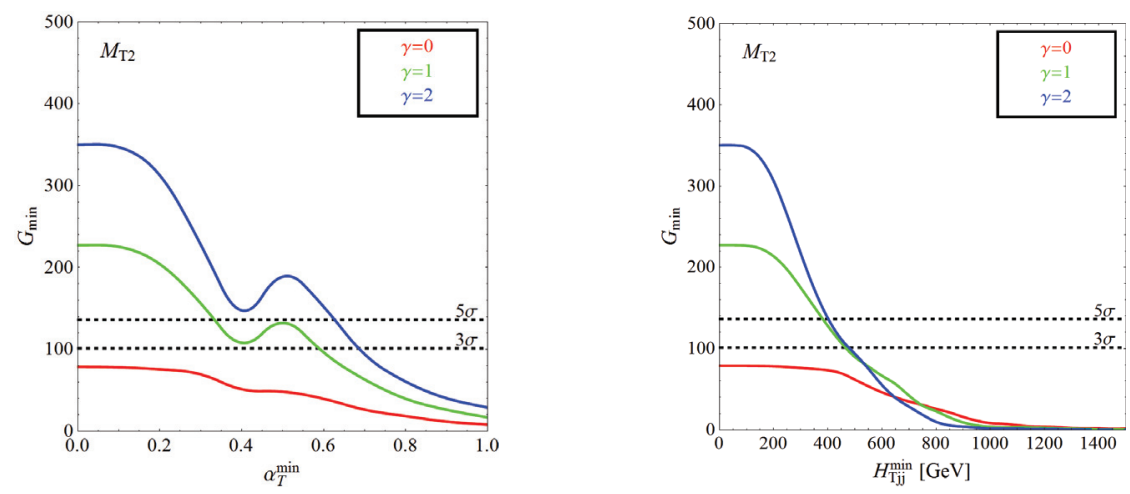

FIGURE 5. The value of the statistic $G_{\min }$ for the $M_{T 2}$ distributions associated with several DDM ensembles as a function of the minimum cut $\alpha_{T}^{\text {min }}$ imposed on $\alpha_{T}$ (left panel) and as a function of the minimum cut $H_{T_{j j}}^{\min }$ imposed on $H_{T_{j j}}$ (right column). In each case, the precuts discussed above are the only other cuts imposed on the data. The curves shown in all panels correspond to the parameter choices $m_{0}=100 \mathrm{GeV}, \Delta m=50 \mathrm{GeV}$, and $\delta=1$, and the $M_{T 2}$ curves correspond to a trial mass $\widetilde{m}=0$.

The procedure we adopt in order to assess the distinctiveness of the $M_{T 2}$ distribution associated with a particular DDM ensemble is similar to the procedure used above. In particular, we canvass the range of traditional dark-matter masses $0<m_{\chi}<m_{\psi}$ with a finite step size, determine the $M_{T 2}$ distribution for each value of $m_{\chi}$ in the survey, compute a goodness-of-fit statistic between this distribution and the $M_{T 2}$ distribution for the DDM ensemble after partitioning both distributions into $n_{b}$ bins, and take the minimum value obtained for this statistic as our final measure of distinctiveness. The only difference is that in this case, we adopt the quantity $G\left(m_{\chi}\right) \equiv-2 \ln \lambda\left(m_{\chi}\right)$, where where $\lambda\left(m_{\chi}\right)$ is the ratio of the likelihood functions for the two distributions, as our goodness-of-fit statistic. Thus, we take

$$
G_{\min } \equiv \min _{m_{\chi}}\left\{G\left(m_{\chi}\right)\right\}
$$

as our final measure of the distinctiveness of the distribution associated with the DDM ensemble. Once again, we estimate the statistical significance for a given value of $G_{\min }$ by comparing this value to a $\chi^{2}$ distribution with $n_{b}-1$ degrees of freedom in order to compute a $p$-value, and then assessing the number of standard deviations away from the mean to which the same $p$-value would correspond for a Gaussian distribution.

In Fig. 5 we show how our measure of distinctiveness $G_{\min }$ depends on the threshold cuts $\alpha_{T}^{\min }$ and $H_{T_{i j}}^{\min }$ imposed on $\alpha_{T}$ and $H_{T_{j j}}$. Once again, the precuts are the only additional cuts imposed on the data. The left panel of the figure shows that while $G_{\min }$ generally decreases with increasing $\alpha_{T}^{\min }$ due to the overall reduction in number of signal events, it does not do so monotonically. Indeed, for all curves shown, $G_{\min }$ actually rises with increasing $\alpha_{T}^{\min }$ within the range $0.4 \lesssim \alpha_{T}^{\min } \lesssim 0.55$ as a result of the advantageous correlations between $\alpha_{T}$ and $M_{T 2}$. By contrast, the corresponding curves in the right panel fall monotonically with $H_{T_{j j}}^{\min }$ due to the loss in statistics. This is to be expected, as we have seen that there is no advantageous correlation between $H_{T_{j j}}$ and $M_{T 2}$ which can be exploited to offset this loss. 


\section{CONCLUSIONS}

Distinguishing between minimal and non-minimal dark sectors at colliders typically involves more that merely identifying an excess in the total number of signal events over background. In particular, it typically requires a detailed analysis of the shapes of relevant kinematic distributions. We have shown that DDM ensembles can give rise to characteristic signatures in such distributions which permit one to distinguish them from traditional dark-matter candidates. Moreover, we have shown that in a variety of cases, such features would provide sufficient evidence to distinguish a DDM ensemble from any traditional dark-matter candidate at the $5 \sigma$ significance level at the upgraded LHC.

It is also important to note that in comparison with simple, "bump-hunting searches," distribution-based searches of this sort involve a number of additional subtleties. For example, cuts imposed on the data for purposes of background reduction can distort event-shape distributions whenever non-trivial correlations exist between the corresponding collider variables. To illustrate the consequences such subtleties, we have examined the effect of these distortions on the event-shape distributions of the kinematic variables most sensitive to the structure of the dark sector in the dijet $+H_{T}$ channel - and in particular the $M_{T 2}$ variable. We have shown that appropriately chosen cuts on particular variables (such as $\alpha_{T}$ ) can actually enhance the distinctiveness of these distributions, while cuts imposed on other variables (such as $H_{T_{j i}}$ ) tend to obscure signals of non-minimality in the dark sector. More details behind all of these results can be found in Refs. [3, 4].

\section{ACKNOWLEDGMENTS}

KRD and BT would like to thank CETUP* for hospitality during the 2015 Dark-Matter Workshop and PPC Conference. BT would also like to thank CETUP* for financial support during the workshop. The research activities of KRD and SS were supported in part by the Department of Energy under Grant DE-FG02-13ER-41976, while the research activities of BT were supported in part by internal funding from Reed College. The research activities of KRD were also supported in part by the National Science Foundation through its employee IR/D program. The opinions and conclusions expressed herein are those of the authors, and do not represent any funding agencies.

\section{REFERENCES}

[1] K. R. Dienes and B. Thomas, Phys. Rev. D85, p. 083523 (2012).

[2] K. R. Dienes and B. Thomas, Phys. Rev. D85, p. 083524 (2012).

[3] K. R. Dienes, S. Su, and B. Thomas, Phys. Rev. D86, p. 054008 (2012).

[4] K. R. Dienes, S. Su, and B. Thomas, Phys. Rev. D91, p. 054002 (2015).

[5] A. Airapetian et al. (ATLAS), (1999), CERN-LHCC-99-14, ATLAS-TDR-14.

[6] V. Khachatryan et al. (CMS), Phys. Rev. Lett. 105, p. 211801 (2010).

[7] S. Chatrchyan et al. (CMS), Phys. Lett. B700, 187-206 (2013).

[8] C. G. Lester and D. J. Summers, Phys. Lett. B463, 99-103 (1999).

[9] V. Khachatryan et al. (CMS), Phys. Lett. B698, 196-218 (2013).

[10] L. Randall and D. Tucker-Smith, Phys. Rev. Lett. 101, p. 221803 (2008).

[11] S. Chatrchyan et al. (CMS), Phys. Rev. Lett. 107, p. 221804 (2011).

[12] S. Chatrchyan et al. (CMS), Eur. Phys. J. C73, p. 2568 (2013).

[13] J. Alwall, M. Herquet, F. Maltoni, O. Mattelaer, and T. Stelzer, JHEP 06, p. 128 (2011).

[14] A. Alloul, N. D. Christensen, C. Degrande, C. Duhr, and B. Fuks, Comput. Phys. Commun. 185, 2250-2300 (2014).

[15] C. Collaboration (CMS), (2011), CMS-PAS-JME-10-014.

[16] C. Collaboration (CMS), (2007), CMS-PAS-JME-07-003.

[17] A. J. Barr, C. G. Lester, M. A. Parker, B. C. Allanach, and P. Richardson, JHEP 03, p. 045 (2003).

[18] A. Barr, C. Lester, and P. Stephens, J. Phys. G29, 2343-2363 (2003).

[19] W. S. Cho, K. Choi, Y. G. Kim, and C. B. Park, Phys. Rev. Lett. 100, p. 171801 (2008).

[20] W. S. Cho, K. Choi, Y. G. Kim, and C. B. Park, JHEP 02, p. 035 (2008).

[21] J. Kumar, A. Rajaraman, and B. Thomas, Phys. Rev. D84, p. 115005 (2011).

[22] G. Aad et al. (ATLAS), Phys. Lett. B701, 186-203 (2013).

[23] W. Beenakker, R. Hopker, M. Spira, and P. M. Zerwas, Nucl. Phys. B492, 51-103 (1997).

[24] W. Beenakker, M. Kramer, T. Plehn, M. Spira, and P. M. Zerwas, Nucl. Phys. B515, 3-14 (1998).

[25] G. Aad et al. (ATLAS), Phys. Lett. B710, 67-85 (2013).

[26] J. Beringer et al. (Particle Data Group), Phys. Rev. D86, p. 010001 (2012). 